\title{
Confinement-induced demixing and crystallization
}

\author{
Gerhard Jung $\odot^{*}$ and Charlotte F. Petersen \\ Institut für Theoretische Physik, Universität Innsbruck, Technikerstraße 21A, A-6020 Innsbruck, Austria
}

(Received 25 March 2020; accepted 20 July 2020; published 6 August 2020)

\begin{abstract}
We simulate a strongly size-disperse hard-sphere fluid confined between two parallel hard walls. We find that confinement induces crystallization into $n$-layered hexagonal lattices and a novel honeycomb-shaped structure, facilitated by fractionation. The onset of freezing prevents the formation of a stable glass phase and occurs at much smaller packing fraction than in bulk. Varying the wall separation triggers solid-to-solid transitions and a systematic change in the size distribution of crystalline particles, which we rationalize using a semiquantitative theory. We show that the crystallization can be exploited in a wedge geometry to demix particles of different sizes.
\end{abstract}

DOI: 10.1103/PhysRevResearch.2.033207

\section{INTRODUCTION}

Confinement occurs naturally in many physical, chemical, and biological systems, such as nanotubes, porous rocks, or crowded living cells. Even for the simplest case of monodisperse hard spheres confined between parallel hard walls one observes spatially inhomogeneous density profiles and diffusivities [1,2], anisotropic structure factors [3,4], multiplereentrant glass transitions [5,6] and solid-to-solid transitions between different crystalline phases [7-10]. Confinement has also been reported to have a strong impact on the structural relaxation of supercooled liquids $[6,11,12]$ since it restricts the range of accessible length scales, which becomes increasingly important as the glass transition is approached [11]. The effect of confinement on the properties of simple liquids or colloids has, therefore, been carefully studied with experiments [13-23], theory [1,3,7,8,24-26], and simulations [2,912,27-34]. The results indicate that the rich phenomenology of confined hard spheres indeed accurately applies to liquids and colloids in nanoscopic confinement. It has also been highlighted that these confinement-induced structural properties can strongly depend on the specifics of the system, such as the roughness of the walls [35] and the interaction between the particles [36].

An important aspect has, however, not yet received much attention, which is the effect of particle size dispersity on the above phenomenology. In laboratory experiments on colloids, a size dispersity occurs naturally from synthesis. Additionally, for the study of structural relaxation in supercooled liquids and glasses a size dispersity must be introduced to prevent crystallization even at very small packing fractions $[6,12,13]$. Despite their popularity as model glass formers,

\footnotetext{
*Corresponding author: gerhard.jung@uibk.ac.at
}

Published by the American Physical Society under the terms of the Creative Commons Attribution 4.0 International license. Further distribution of this work must maintain attribution to the author(s) and the published article's title, journal citation, and DOI. recent observations in bulk have revealed that Gaussiandistributed hard spheres [37-40] as well as the often used Kob-Anderson model $[41,42]$ form crystals already in the supercooled regime. This crystallization is induced by a process called fractionation which describes the separation of a homogeneous fluid into different liquid or crystalline fractions with very different particle-size distributions. Since walls or other boundaries lead to heterogeneous crystallization which is known to strongly effect and often enhance nucleation processes $[43,44]$, it should be expected that confinement could also have an impact on crystallization and fractionation. Understanding the equilibrium structural properties of confined size-disperse particles is thus a critical outstanding problem and is essential for future studies of simple glass formers and colloids in confinement.

In this paper, we study the crystallization of size-disperse hard spheres in a slit geometry. We use two complementary approaches: First, we perform exact event-driven molecular dynamics (EDMD) simulations with enhanced sampling techniques to reveal a complex phase behavior, displaying, among others, a new phase which is shaped like a honeycomb lattice. Significantly, we find formation of stable crystals at much smaller packing fractions than reported for bulk systems and show that the crystallization is enabled by a confinementcontrolled fractionation. These surprising results show additionally that the multiple-reentrant glass transition reported in Ref. [6] is only metastable. Second, we rationalize our findings with a semiquantitative theory allowing us to provide a deep insight into the mechanisms that drive the confinementinduced crystallization. Most importantly, our study reveals a very general technique for the demixing of size-disperse particles with excluded volume interactions.

\section{METHODS}

The investigated system consists of two parallel hard walls separated by a distance $H$ in the $z$ direction and periodic boundary conditions in the other two dimensions. The slab is filled with size-disperse hard spheres at packing fraction $\phi$. In the spirit of recent simulations in bulk [40], we employ 
a Gaussian distribution with average particle diameter $\sigma$ and dispersity $\delta=0.15$. Here, $\sigma$ sets the length scale of the system, and the dispersity $\delta$ is defined as the ratio of the standard deviation of the particle diameter to the average particle diameter.

\section{A. Event-driven molecular dynamics simulations}

The system is simulated using a hybrid scheme of EDMD simulations in the $N V T$ ensemble [45-47] and the recently proposed swap Monte Carlo algorithm (SWAP) [48-50]. SWAP introduces "swap events" that are triggered every $\Delta t=$ $0.5 \sigma v_{\text {th }}^{-1}$ time steps. Here, the thermal velocity $v_{\text {th }}=\sqrt{k_{\mathrm{B}} T / m}$ sets the unit of time $\sigma v_{\text {th }}^{-1}$ with particle mass $m$, temperature $T$, and Boltzmann's constant $k_{B}$. The thermal energy $k_{B} T$ sets the unit of energy. Each swap event consists of $N_{s}=50000$ Monte Carlo moves in which two particles are randomly selected and their diameters are exchanged. The move is accepted if the particles exhibit no overlap with other particles after the swap. This corresponds to the typical Monte Carlo acceptance criterium for hard spheres and is consistent with detailed balance. The hybrid scheme is implemented in the EDMD code DYNAMO [47]. Its usage is essential to accelerate the dynamics and systematically ensure that the equilibrium state of the system is attained. We observe typical acceptance ratios of $\Gamma=0.05$, showing that a significant proportion of the particles is swapped in each swap event. The slab consists of $N=13068$ particles for any wall separation $2.0 \leqslant H / \sigma \leqslant$ 3.0 resulting in a minimum longitudinal box size of $L_{x}=L_{y}=$ $67.15 \sigma$, which is large enough to eliminate any finite-size effects. The system is equilibrated for $N_{\mathrm{e}}=3 \times 10^{10}$ events, which roughly corresponds to a total equilibration time of $t_{\mathrm{eq}}=7.0 \times 10^{4} \sigma v_{\mathrm{th}}^{-1}$.

To determine the size distribution of particles in the solid phase, we define the rotationally invariant bond-order parameter $d_{6}(m, n)=\phi_{6, m} \phi_{6, n}$. Here, we introduced the local bondorientational parameter, $\phi_{6, m}=N_{m}^{-1} \sum_{m^{\prime}=1}^{N_{m}} e^{6 \mathrm{i} \theta_{m m^{\prime}}}$ as the relative orientation of particle $m$ with its $N_{m}$ neighbors [51-53]. Neighbors are all particles $m^{\prime}$ within a cutoff distance $r_{\|}<$ $1.4 \sigma$ and $r_{\perp}<0.1 \sigma$ with $\perp$ denoting the direction perpendicular to the walls. Using these invariant measures, we define a particle $m$ as crystalline if it has at least four neighbors $n$ for which the rotationally invariant bond-order parameter $d_{6}(m, n)>0.7$. Above this threshold, the local environment of particle $m$ is strongly correlated with the one of neighbor $n$ which indicates a solidlike structure. To identify the big particles in the honeycomb phase $3 \odot$, we set $r_{\|}<1.8 \sigma$ and require only three crystalline neighbors. The threshold values were selected by investigating individual configurations by eye and ensuring that the crystals are correctly identified. We also verify that our results do not depend crucially on the precise threshold values. This order parameter can clearly only detect crystals with hexagonal order, and it cannot differentiate between crystals with different numbers of lattice planes. We report the packing fraction $\phi_{c}$ at which the onset of crystallization is observed as well as the average particle diameter $\bar{a}$ and dispersity $\delta_{c}$ of particles in the solid phase [51-53]. The observables are averaged over all crystalline particles for several independent runs for packing fractions $\phi_{c} \lesssim \phi<\phi_{c}+0.03$. We verify for every configuration that there is no systematic dependence of these observables on the packing fraction. The variation between independent runs is less than $2 \%$.

\section{B. Theoretical model}

The theoretical approach generalizes the technique proposed for monodisperse particles [8] by combining fundamental measure theory for size-disperse hard spheres in a slit geometry [54-56] with cell theory [57-59].

\section{Fundamental measure theory}

Fundamental measure theory (FMT) is based on density functional theory and minimizes the functional for the grand free energy leading to the equation $[54,55]$,

$$
\ln \lambda_{i}^{3} n_{i}(z)=\beta \mu_{i}-\beta \frac{\delta \mathcal{F}^{\mathrm{ex}}\left[n_{i}\right]}{\delta n_{i}(z)}-\beta V_{i}(z) .
$$

Here, $n_{i}(z)$ is the local number density, $\mu_{i}$ is the chemical potential [56], and $V_{i}(z)$ is the external wall potential of component $i$. Each component has a different diameter $a_{i}=a_{\min }+(i+0.5) \Delta a$, which allows us to emulate a size dispersity by creating a mixture of $m$ different components, similar to Ref. [6]. We use $m=25$ with $\Delta a=0.024 \sigma$ and $a_{\min }=0.7 \sigma$. We also ensured that the density profiles and the resulting free energies only marginally change $(\Delta \mathcal{F}<$ $0.02 k_{B} T$ ) upon an increase in $m$. For the excess free-energy functional of a hard-sphere mixture $\mathcal{F}^{\text {ex }}$ we use the White Bear Version II [55]. To determine the density profile $n_{i}(z)$, Eq. (1) is solved self-consistently in an iterative procedure [55]. Different from Ref. [6], we ensure in our algorithm that the chemical potential $\mu_{i}$ is adapted in each iteration such that the resulting slit-averaged concentrations of every component $\bar{n}_{i}\left(a_{i}\right)=H^{-1} \int d z n_{i}(z)$ precisely match a Gaussian profile with mean $\sigma$ and variance $\delta$. The free energy per particle of component $i$ can then be evaluated from its ideal and excess contributions,

$$
\mathcal{F}_{\mathrm{lq}, i}=\frac{1}{\beta H \bar{n}_{i}} \int d z n_{i}(z)\left[\ln \lambda_{i}^{3} n_{i}(z)-1\right]+\frac{\mathcal{F}^{\mathrm{ex}}}{N} .
$$

The constant $\lambda_{i}$ is set to $\lambda_{i}^{3}=\sigma^{4} \Delta a^{-1}$ such that $\rho_{i}(z)=$ $\lambda_{i}^{3} n_{i}(z)=\sigma^{4} \Delta a^{-1} n_{i}(z)$ is the density distribution of the disperse mixture in the spirit of Ref. [37]. We carefully checked this expression and ensured that it is independent of $a_{\min }$ and $\Delta a$ and, indeed, gives the correct ideal gas contributions (e.g., the expected values for the mixing free energy).

The results for the free energy per particle of a hard-sphere mixture in the liquid state are shown in Fig. 1. It can be observed that the maximum of the distribution is shifted to around $1.07 \sigma$. This is because the accessible width of the slab for hard spheres is $L=H-a$, which means that the density of big particles (with large $a$ and, thus, small $L$ ) in the accessible region is higher than the one of the small particles. This similarly holds for the ideal free energy in the liquid phase $\mathcal{F}_{\mathrm{lq}}(a)$. Furthermore, there is a pronounced nonmonotonic dependence of the free energy on the wall separation $H$. In Refs. [4,6], a similar nonmonotonic behavior in the structure factor was explained with commensurate and incommensurate packings. Commensurate packing describes the favorable packing of spheres in $k$ layers for wall separations $H / \sigma \approx k, k \in \mathbb{N}$, whereas incommensurate 


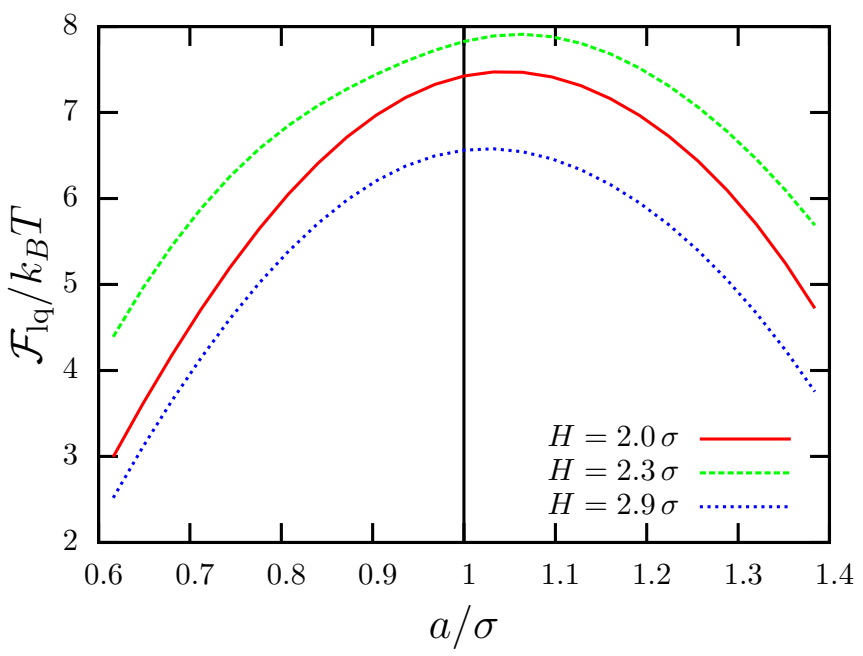

FIG. 1. Free energy per particle $\mathcal{F}_{\mathrm{lq}}$ of a hard-sphere fluid in the liquid state for particles with different diameters $a$ in a slab with wall separation $H$ and packing fraction $\phi=0.50$. The vertical black line corresponds to the maximum of the host fluid density distribution at $a=\sigma$.

packing relates to wall separations close to half-integer values. The latter is less favorable since single particles have to be included between the $k$ layers which also leads to a slowing down of diffusion.

\section{Cell theory}

To determine the free energy of particles in the crystalline phase, we employ cell theory [8,57-59]. As input, we chose five different lattice types $(2 \triangle, 3 \triangle, 3 \odot$, as well as the square lattices $2 \square$ and $3 \square$ ) which can be described by two or three lattice parameters $c_{i}$. One of these parameters is fixed to set the desired packing fraction $\phi$. For each lattice type $C$, we create a minimal structure of the crystals in a slab geometry with wall separation $H$ and packing fraction $\phi$ and place particles on the lattice sites with diameter $\bar{a}$. Only one of the lattice sites is occupied with a test particle of diameter $a$. For this particle, we determine the free volume cell using a Monte Carlo procedure and thus determine its free energy based on the assumptions used in cell theory. For each $H, \phi, \bar{a}$, and $a$, we minimize this free energy with respect to the free lattice parameters $c_{i}$. In crystals with three layers, there is a difference in the free volume cell of particles in the center of the lattice or at the wall. In this case, we determine the contributions separately and minimize the averaged free energy per particle.

\section{Determination of the stability diagram}

Having determined the free energies in the liquid phase, $F_{\mathrm{lq}}(a \mid H, \phi)$, and the crystalline phase, $\mathcal{F}_{\mathrm{c}}(a \mid H, \phi, C, \bar{a})$, we assume a top-hat distribution $f\left(a \mid \bar{a}, \delta_{c}\right)$ of particles in the crystalline phase with size-dispersity $\delta_{c}$ and create a list of free-energy differences,

$$
\begin{aligned}
\Delta \mathcal{F}\left(H, \phi, C, \bar{a}, \delta_{c}\right)= & \Delta \mathcal{F}_{\text {mix }}\left(\delta_{c}\right)+\Delta \mathcal{F}_{\text {shift }}+\int d a f\left(a \mid \bar{a}, \delta_{c}\right) \\
& \times\left[\mathcal{F}_{\mathrm{c}}(a \mid H, \phi, C, \bar{a})-\mathcal{F}_{\mathrm{lq}}(a \mid H, \phi)\right] .(3)
\end{aligned}
$$

Here, we have to account explicitly for the mixing freeenergy $\Delta \mathcal{F}_{\text {mix }}\left(\delta_{c}\right)=-\ln \left(\sqrt{12} \delta_{c}\right)$ of the particles in the crystal $[60,61]$ and include a shift parameter $\Delta \mathcal{F}_{\text {shift }}$ since cell theory only provides an upper bound for the free energy. The latter was set to $\Delta \mathcal{F}_{\text {shift }}=-1.80 k_{B} T$ in Ref. [8] to match the value for monodisperse disks in the two-dimensional limit. We adopted this value in our paper. For every wall separation $H$, we report the minimal packing fraction $\phi_{c}$ for which the liquid and crystalline free energies are equal $\Delta \mathcal{F}=0$. Additionally, we determine the corresponding crystalline-type $C$, the mean particle diameter $\bar{a}$, and dispersity $\delta_{c}$.

In a monodisperse system, a coexistence region of liquid and crystalline phases can be determined from the free energies using the common tangent construction. The underlying assumption is that, inside the coexistence region, both the liquid and the crystalline phases have a constant packing fraction (and obviously a constant particle-size distribution). The fractionation in size-disperse systems, however, implies that the particle-size distribution in the liquid phase changes with the onset of crystallization. In Refs. [37,62], it was shown that these systems can be handled using the moment freeenergy method, leading to highly coupled nonlinear equations. In the case of confinement, the application of this method is unfeasible since the free energies can only be determined numerically, and it is not clear how they could be rewritten such that they only depend on four moments of the (positiondependent) density distribution. Here, we thus rely on a semiquantitative scheme and identify the critical packing fraction $\phi_{c}$ in a system with wall separation $H$ as the packing fraction at which the free energies in the crystalline and the liquid phases are equal.

\section{STABILITY DIAGRAM IN SLAB GEOMETRIES}

We first study the crystallization of hard spheres confined in slabs with different wall separations $2.0<H / \sigma<3.0$. The system displays an onset of crystallization at packing fractions around $\phi \approx 0.5$, much smaller than the bulk value $\phi_{b} \approx$ 0.6 [40]. Four different crystalline phases can be observed, namely, an amorphous liquid phase for small packing fraction $\phi$, a two-layered hexagonal structure $(2 \triangle$ ) for small $H$, a three-layered hexagonal structure $(3 \triangle)$ for large $H$, and an intermediate honeycomb-shaped phase $(3 \odot)$. A visualization of the different phases is shown in Fig. 2, the stability diagram is displayed in Fig. 3. The sequence of solid-to-solid transitions $2 \triangle \rightarrow 3 \odot \rightarrow 3 \triangle$ upon a change in wall separation is reminiscent of similar transitions in the case of monodisperse hard spheres or charged colloids [8,63,64]. Different from these monodisperse systems, the size dispersity leads to a significantly enlarged domain where the hexagonal structures are stable. In the small intermediate domain between $2 \triangle$ and $3 \triangle$, we observe the honeycomb phase, which consists of three layers of small particles forming honeycomb cells and big particles in the upper and lower layers which fill these cells, visualized in Fig. $2(3 \odot)$. The formation of this honeycomb structure leads to the suppression of a square-lattice phase which was observed in the case of small or vanishing dispersity $[8,12]$. In fact, for $\delta=0.15$, the square-lattice is only stable in simulations at very high packing fraction $\phi>0.53$ in coexistence with a liquid and a honeycomb phase. 


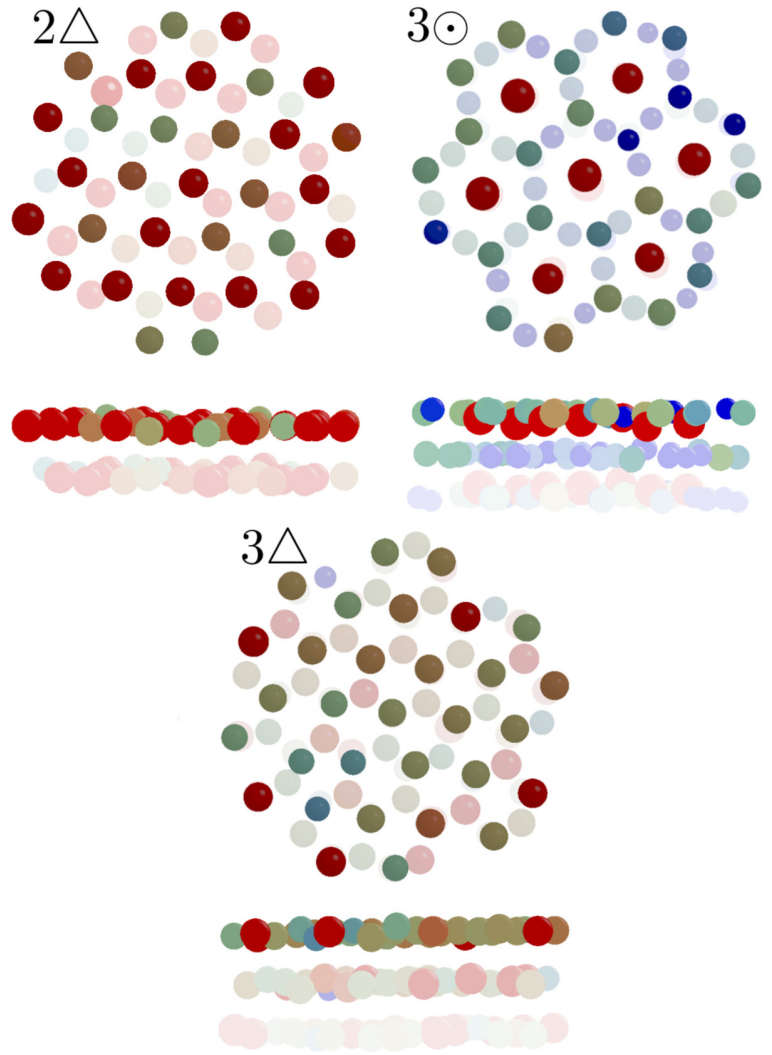

FIG. 2. Visualization of the different phases observed in the slab geometry. $(2 \triangle)$ Two hexagonal layers for $H=2.2 \sigma, \phi=$

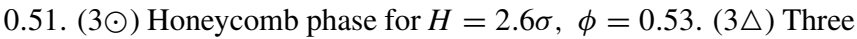
hexagonal layers for $H=3.0 \sigma, \phi=0.53$. Color code indicates particle diameter with $a<0.9 \sigma$ (blue), $a>1.1 \sigma$ (red), and interpolation between. The sphere diameter is scaled by a factor of 0.5 , and the lower layers are partially transparent for the sake of visualization.

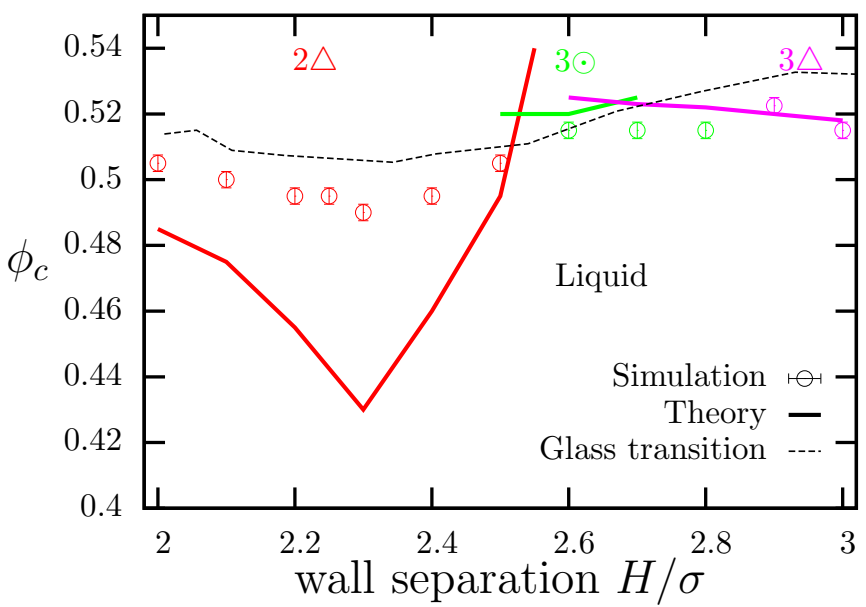

FIG. 3. Stability diagram of size-disperse hard spheres confined between two parallel flat walls a distance $H$ apart. Lines show theoretical results, and symbols show simulation results for the critical packing fraction $\phi_{c}$ of the liquid-to-crystal transition. Dashed line corresponds to the glass transition line of the same system as determined in Ref. [6]. Colors indicate transitions to different crystalline phases: Two hexagonal layers $(2 \triangle$, red), honeycomb phase $(3 \odot$, green), and three hexagonal layers $(3 \triangle$, pink).

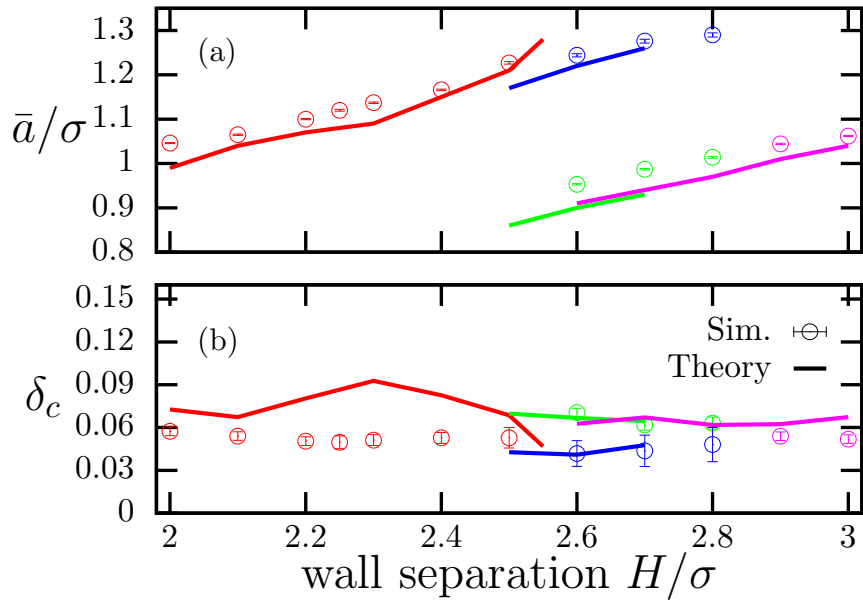

FIG. 4. (a) Average particle diameter $\bar{a}$ and (b) dispersity $\delta_{c}$ of particles in the different crystalline phases at packing fraction $\phi \gtrsim \phi_{c}$. Lines show theoretical results and symbols show simulation results. Colors indicate the different crystalline phases: $2 \triangle$ (red), $3 \odot$ (small particles: green, big particles: blue), and $3 \triangle$ (pink).

Comparing the critical packing fractions presented in Fig. 3 to the glass transition line determined in Ref. [6] shows that the glass is metastable and can only coexist with a crystalline phase. Interestingly, both the glass transition line and the critical packing fraction $\phi_{c}$ exhibit a pronounced nonmonotonicity leading to the possibility of observing reentrant phase transitions by changing the wall separation $[6,65]$.

The size distribution of particles in the emergent crystals at packing fraction $\phi \gtrsim \phi_{c}$ is strongly correlated with the wall separation $H$ as shown in Fig. 4(a). The average particle diameter $\bar{a}$ for crystalline particles exhibits a linear dependence on $H$ for one crystalline phase and a distinct jump upon a solid-to-solid transition. It can also be observed that the honeycomb phase is indeed interpolating between the two hexagonal structures since the diameters of the big and small particles in the honeycomb phase perfectly follow the linear dependence of the $2 \Delta$ and $3 \Delta$ hexagonal phases, respectively. The size dispersity of the crystalline particles is around $\delta_{c} \approx 0.05-0.06$, much smaller than the dispersity of the host fluid $\delta=0.15$, which indicates a significant fractionation [see Fig. 4(b)]. The dispersity of crystalline particles in confinement is very close to the one for crystals in bulk $\delta_{c} \lesssim 0.07$, and similar for all of the crystalline phases we observed.

A major impact of size dispersity on the stability diagram is the enlargement of domains with stable hexagonal structures compared to the monodiperse case. This is facilitated by a linear increase in the average diameter $\bar{a}$ with wall separation $H$, seen in Fig. 4(a), leading to a constant ratio $H / \bar{a}$. Consequently, in the extreme case of a flat size distribution of the host fluid, $H$ would reduce to a mere length scale setting the average particle size $\bar{a}$, leading to the existence of just one crystalline phase and a constant critical packing fraction $\phi_{c}$. In reality, however, this picture is oversimplified since the particle-size distribution of the host fluid already defines the length scale $\sigma$. Therefore, the values of $\bar{a}$ are bound to $\sigma(1-\delta) \lesssim \bar{a} \lesssim \sigma(1+\delta)$. Upon a further increase in $H$ (and 
thus $\bar{a}$ ), a discontinuous solid-to-solid transition is triggered by increasing the number of crystalline layers and reducing the average diameter of crystalline particles.

We additionally expect that the maximum in the particlesize distribution of the host fluid would lead to the existence of a minimum in $\phi_{c}$ which implies a reentrant melting transition. The minimum should be approximately located at a wall separation $H$ for which the average particle diameter $\bar{a}=\sigma$. Comparing Figs. 3 and 4(b), however, shows that this minimum is shifted and placed around $\bar{a}=1.1 \sigma$. To rationalize this observation, we consider the free-energy distribution $\mathcal{F}_{\mathrm{lq}}(a)$ calculated with FMT, which indeed exhibits a shifted maximum around $a \approx 1.07 \sigma$ as discussed in Sec. II B. Additionally, the differences between commensurate and incommensurate packings in the slab leads to an increase in the liquid free energy for $H \approx 2.5 \sigma$ (see Fig. 1 ).

Using the semiquantitative theory, we can, therefore, explain the emergence and location of the reentrant melting transition. The depth of the minimum in the critical packing fraction $\phi_{c}$ is, however, exaggerated in the theory, which also manifests itself in deviations from the expected dispersity in Fig. 4(b). That being said, the theory shows a near-quantitative agreement with the simulation results for all investigated quantities.

This analysis shows that the crystallization of disperse particles in confinement relies on fractionation, similar to previous observations in bulk $[37,40]$. In both cases, we would expect that the free-energy barriers that have to be overcome for the particles to diffuse and order will be significantly larger compared to the case of monodisperse crystallization, which could lead to long nucleation times. For high dispersity, this is likely to reach timescales much longer than typical laboratory experiments. In this paper, we significantly accelerated the equilibration and crystallization by using the SWAP algorithm as discussed in Sec. II A. Without the use of this algorithm, nucleation would be too slow to observe crystallization within a reasonable computing time [4,6]. However, the onset of ordering can already be seen in standard EDMD simulations of hard spheres in confinement (see the long-wavelength peak in the structure factor for $H=2.30 \bar{\sigma}$ shown in Ref. [4]).

\section{CONFINEMENT-CONTROLLED FRACTIONATION IN A WEDGE GEOMETRY}

The systematic dependence of fractionation on wall separation revealed in the previous section can be used to order particles by their size. We highlight this by tilting one of the walls by a small angle $\alpha=1^{\circ}$, thus creating a wedge geometry with a linear height profile $H(x)=H_{0}+\tan (\alpha) x$ as shown in Fig. 5(a) $[6,64]$. The wedge is closed at $H(x)=4.0 \sigma$ by a vertical flat wall, and only the $y$ direction is periodic with $L_{y}=60 \sigma$. The wedge consists of 25000 particles leading to an average packing fraction $\bar{\phi}=0.51$. We use $N_{s}=100000$ Monte Carlo moves per swap event and employ a similar equilibration time as used in the slab geometry. For the purpose of evaluation, the wedge is separated into slices of size $\Delta H=0.05 \sigma$, and local averages are calculated to determine the profiles shown in Fig. 5. We perform simulations for four independent wedges per tilt angle $\alpha$ to estimate the uncertainty of the presented profiles. (a)
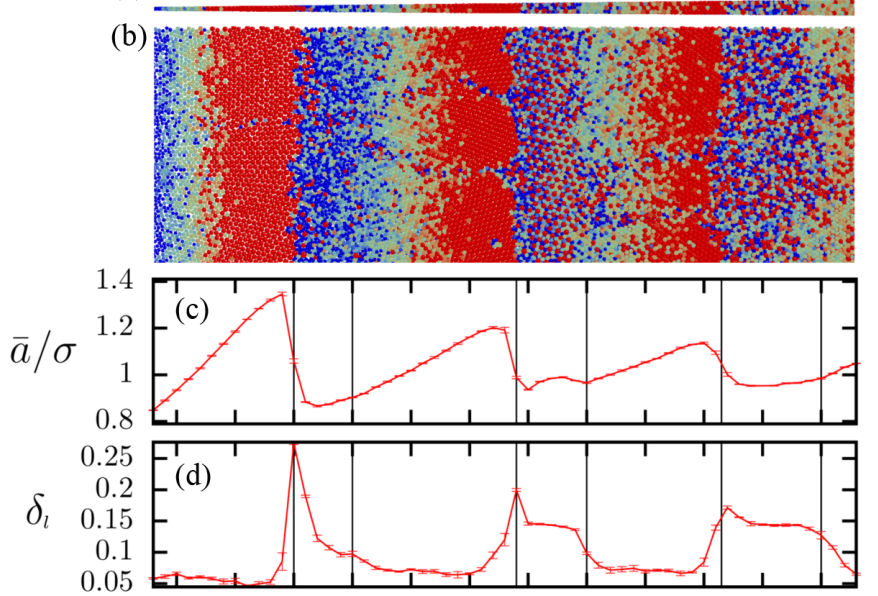

$N_{c}$
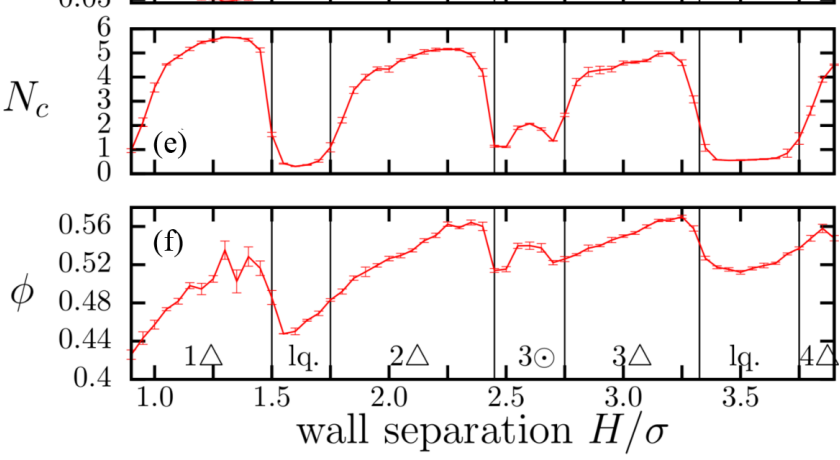

FIG. 5. Demixing in a wedge geometry with average packing fraction $\bar{\phi}=0.51$. Upper panels show snapshots of a typical configuration in (a) side view and (b) top view. The color code is the same as used in Fig. 2. (c)-(f) Show local averages of mean particle diameter $\bar{a}$, local dispersity $\delta_{l}$, number of crystalline neighbors per particle $N_{\mathrm{c}}$, and packing fraction $\phi$. The averages and errors were determined from four independent runs of the same system. The black vertical lines mark the transition between different phases and the labels in panel (f) indicate their respective lattice types or the liquid phase (lq.).

After equilibration, separate liquid and crystal regions are observed in the wedge, displaying the same phase behavior as discussed for the slab geometry (compare Figs. 3 and 5). We find that the average particle diameter $\bar{a}$ exhibits a pronounced sawtoothlike dependence on the local wall separation $H(x)$ whereas the dispersity attains values between $\delta_{l} \approx$ 0.05 and 0.07 in the crystalline regions (number of crystalline neighbors $N_{c}>3.0$ ) and $\delta_{l} \approx 0.1-0.15$ in the liquid regions $\left(N_{c}<1.0\right)$ [see Figs. 5(c)-5(e)].

We also observe large local variations in the packing fraction $\phi(H)$ with wall separation both in the liquid and in the crystalline regions [see Fig. 5(f)]. Two very different factors lead to these local density variations. First, even at low average packing fraction in a pure liquid, oscillations in the local packing emerge due to the layering of the particles [6], which explains the behavior of $\phi(H)$ in the liquid regions. Second, the local variation of the average particle diameter $\bar{a}(H)$ in the crystalline regions leads to mechanical stress in the crystals. Assuming a uniform lattice constant, we could expect a quadratic increase in the packing fraction $\phi(H)$ since $\bar{a}$ scales with $H$. However, a close inspection of the snapshot 
in Fig. 5(b) reveals that the system responds to the mechanical stress by bending the lattice planes (see Appendix B), which significantly reduces the variation in packing fraction. As a consequence, for small $\bar{a}$, the packing fraction increases approximately linearly in the crystalline regions, and it is nearly constant for large $\bar{a}$.

We have verified that the above results are mostly independent of the tilt angle $\alpha$. The only noticeable difference is the suppression of the honeycomb phase when significantly increasing the tilt angle $\left(\alpha>2^{\circ}\right)$. The reason for this is that the region in which this phase would self-assemble becomes too small to create stable crystals. To check the sensitivity of our results to the choice of ensemble, we also performed simulations which included a liquid reservoir adjacent to the wedge but found no significant change to any of the quantities measured in Fig. 5.

\section{CONCLUSION}

We have studied the crystallization of size-disperse hard spheres in a confined geometry. The simulation results uncover solid-to-solid transitions between different crystalline phases and a nonmonotonic dependence of the critical packing fraction for the liquid-to-solid transition. The latter implies the existence of a reentrant melting scenario. Moreover, we have revealed a systematic dependence of the particle-size distribution on the wall separation in the crystalline phase. To rationalize theses findings we have presented a semiquantitative theory with which we can explain all features of the stability diagram and the emergent particle-size distributions.

Due to the universality of the underlying mechanisms which lead to the described crystallization in confinement, we expect that our results generally describe the phase behavior of particles for which excluded-volume interactions dominate. We anticipate that this holds, in particular, for the confinement-controlled demixing and fractionation. We have, therefore, devised a generally applicable technique for the purification of size-disperse particles by insertion into a wedge geometry. The observed fractionation is particularly important for applications involving external shear stresses. It has been widely observed that shear induces fractionation in channels even at moderate packing fractions, which is, for example, an important phenomenon for food processing [66]. We expect that similar fractionation effects arise in dense packings, through a combination of shear-induced crystallization [67] and the confinement-induced fractionation detailed here.

In the future, it would be interesting to extend our studies to laboratory experiments on hard-sphere-like colloids [68,69]. The usage of softer particles might be necessary to overcome the expected large nucleation times, since it is expected that soft repulsion will significantly lower the critical packing fraction while maintaining a reasonable high diffusion rate. Potential candidates are nanocrystals as discussed in Refs. [40,70] or charged colloids. Clearly, softness will weaken the effect of size dispersity, so we expect fractionation to be observable only for larger dispersities. Furthermore, it would be exciting to analyze the influence of external shear stresses or different boundary conditions on the reported crystallization behavior. For example, it is expected that rough walls would
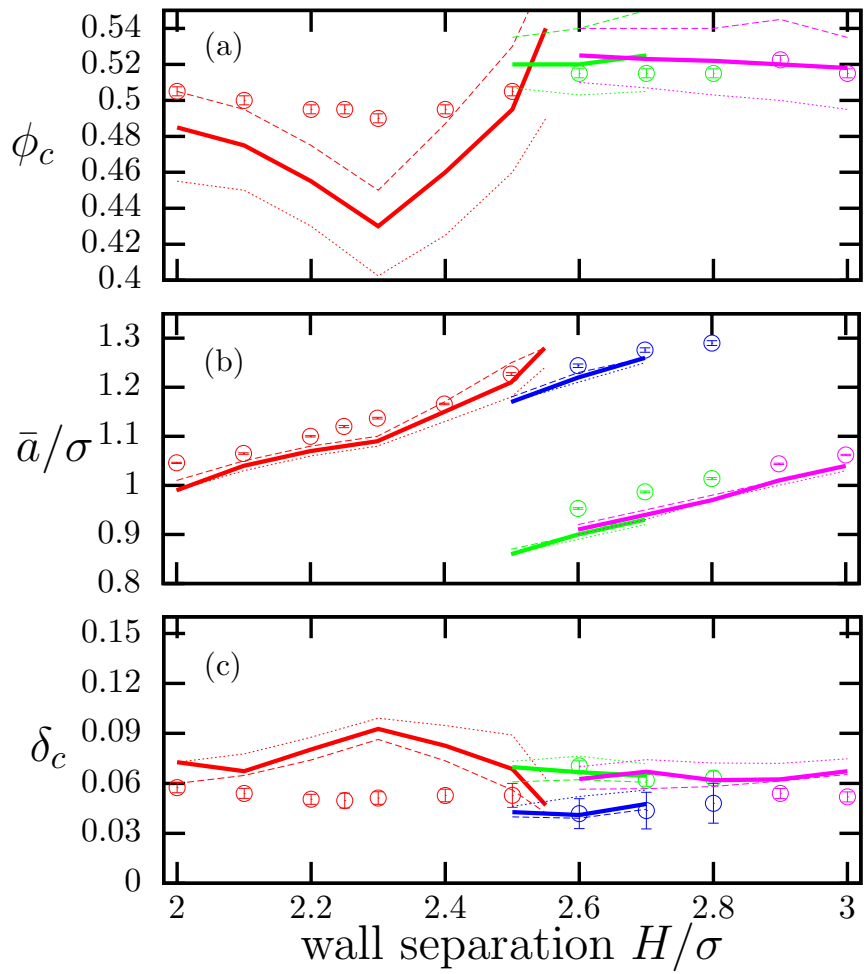

FIG. 6. The same as Figs. 3 and 4 with additional lines for the error estimation of the theory. The dashed/dotted line correspond to an equivalent theory with slightly increased/decreased crystalline free energy per particle $\Delta \mathcal{F}_{\text {shift }}=(-1.80 \pm 0.25) k_{B} T$.

significantly increase the critical packing fraction of the liquid-to-solid transition and should thus enable the existence of a stable glass phase.

\section{ACKNOWLEDGMENTS}

We acknowledge helpful discussions with M. Schmidt, $\mathrm{H}$. Löwen, R. Wittmann, S. Mandal, F. Schmid, and T. Franosch. G.J. was supported by the Austrian Science Fund (FWF) Grant No. I 2887. C.F.P. was supported by the FWF Grant No. M 2471.

\section{APPENDIX A: THEORETICAL MODEL: UNCERTAINTIES}

Additional to the data shown in Sec. III, the theory was also evaluated with shifted free-energy differences $\Delta \mathcal{F}_{\text {shift }}=$ $(-1.80 \pm 0.25) k_{B} T$ to estimate uncertainties originating from the assumptions behind cell theory (see Fig. 6). It should be noted that the value of $0.25 k_{B} T$ is a lower estimate of uncertainties in the cell theory and that systematic errors can obviously not be discussed quantitatively. The most important conclusion from this analysis is that, while a small shift of the free energy leads to quantitative differences, especially for the critical packing fraction, the qualitative features stay the same. It also indicates that the exaggeration of the minimum 


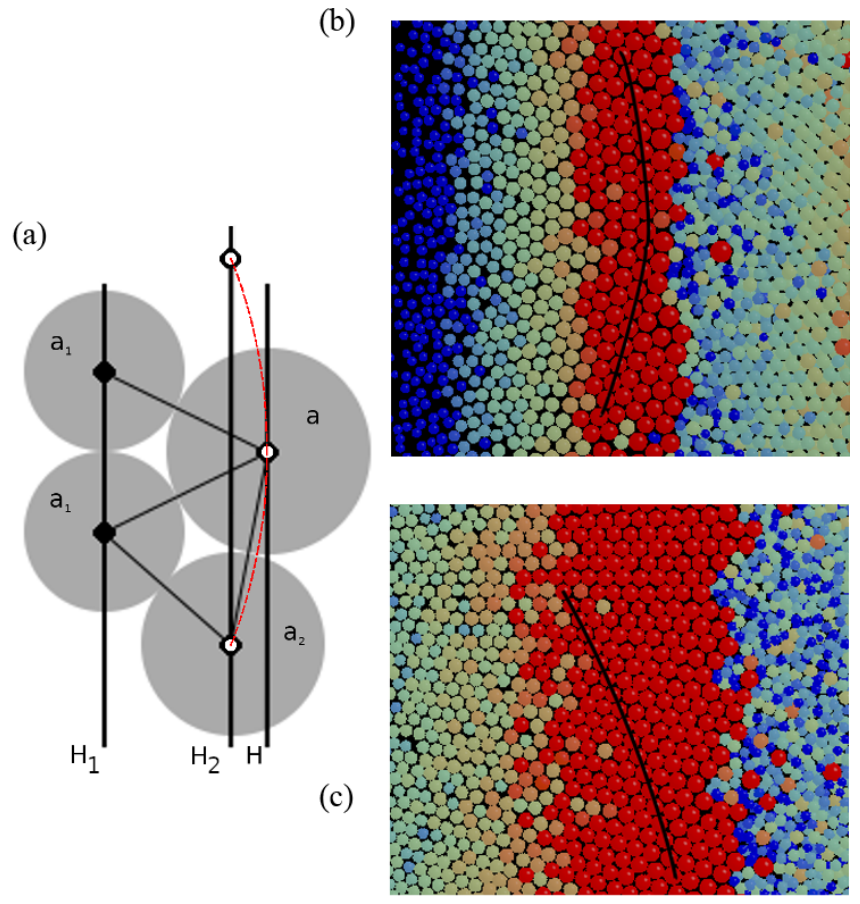

FIG. 7. Curvature of the lattice planes in a wedge geometry. (a) Shows a sketch of how curvature is introduced in a crystal due to local variations in the height $H_{i}$ and thus the diameter $a_{i}$ of the particles. The red dashed line corresponds to the circular sector with radius of curvature $R$. (b) and (c) show snapshots of EDMD simulations in wedge geometries with tilt angle $\alpha=0.044 \mathrm{rad}\left(2.6^{\circ}\right)$ and $\alpha=0.017 \mathrm{rad}\left(1^{\circ}\right)$, respectively. The black lines highlight the curved lattice planes. in the critical packing fraction (and the emergent maximum in the dispersity) is a systematic error of the fundamental measure theory. This error can also be revealed when comparing the density profile $n(z)$ predicted by FMT to eventdriven computer simulations as has already been discussed in Ref. [6]. Here, we find that these deviations remain even without any differences in the setting up of the dispersity. We conclude that strong inhomogeneous density profiles due to broken translational symmetry are not perfectly described by FMT and lead to an overestimation of the free energy for incommensurate packing and high packing fraction.

\section{APPENDIX B: CURVATURE OF THE LATTICE PLANES IN A WEDGE GEOMETRY}

We also discussed, in Sec. IV, the role of local variations in particle diameter $\bar{a}(H)$ inside the crystalline phases which requires the lattice planes to exhibit a certain curvature. For a one-layered hexagonal structure under the mild assumption that $\bar{a}(x)=H(x)$, we can derive from straightforward geometrical calculations a radius of curvature $R$ for lattice planes in the crystalline structures. The sketch in Fig. 7(a) highlights all necessary quantities and a circular sector with the desired radius of curvature $R$. We find

$$
R \approx 1.1 \frac{H}{\alpha(\mathrm{rad})}
$$

From this, we can estimate the radii of curvature $R_{\alpha}$ for the lattice planes marked in Figs. 7(b) and 7(c) under the assumption that $H$ is approximately constant and find $R_{\alpha=1} \approx 70 \sigma$ and $R_{\alpha=2.6^{\circ}} \approx 30 \sigma$ in very good agreement with the measured values of $R_{\alpha=1^{\circ}} \approx 75.4 \sigma$ and $R_{\alpha=2.6^{\circ}} \approx 29.2 \sigma$, respectively.
[1] R. Kjellander and S. Sarman, Chem. Phys. Lett. 149, 102 (1988).

[2] J. Mittal, T. M. Truskett, J. R. Errington, and G. Hummer, Phys. Rev. Lett. 100, 145901 (2008).

[3] B. Götzelmann and S. Dietrich, Phys. Rev. E 55, 2993 (1997).

[4] S. Mandal, S. Lang, V. Boţan, and T. Franosch, Soft Matter 13, 6167 (2017).

[5] S. Lang, V. Botan, M. Oettel, D. Hajnal, T. Franosch, and R. Schilling, Phys. Rev. Lett. 105, 125701 (2010).

[6] S. Mandal, S. Lang, M. Gross, M. Oettel, D. Raabe, T. Franosch, and F. Varnik, Nat. Commun. 5, 4435 (2014).

[7] M. Schmidt and H. Löwen, Phys. Rev. Lett. 76, 4552 (1996).

[8] M. Schmidt and H. Löwen, Phys. Rev. E 55, 7228 (1997).

[9] A. Fortini and M. Dijkstra, J. Phys.: Condens. Matter 18, L371 (2006).

[10] E. C. Oguz, M. Marechal, F. Ramiro-Manzano, I. Rodriguez, R. Messina, F. J. Meseguer, and H. Löwen, Phys. Rev. Lett. 109, 218301 (2012).

[11] P. Scheidler, W. Kob, and K. Binder, Europhys. Lett. 59, 701 (2002).

[12] R. C. Roberts, N. Marioni, J. C. Palmer, and J. C. Conrad, Mol. Phys. 118, e1728407 (2020).

[13] C. R. Nugent, K. V. Edmond, H. N. Patel, and E. R. Weeks, Phys. Rev. Lett. 99, 025702 (2007).
[14] P. S. Sarangapani, A. B. Schofield, and Y. Zhu, Phys. Rev. E 83, 030502(R) (2011).

[15] A. Reinmüller, E. C. Oğuz, R. Messina, H. Löwen, H. J. Schöpe, and T. Palberg, J. Chem. Phys. 136, 164505 (2012).

[16] K. Nygård, R. Kjellander, S. Sarman, S. Chodankar, E. Perret, J. Buitenhuis, and J. F. van der Veen, Phys. Rev. Lett. 108, 037802 (2012).

[17] K. Nygård, S. Sarman, and R. Kjellander, J. Chem. Phys. 139 , 164701 (2013).

[18] G. L. Hunter, K. V. Edmond, and E. R. Weeks, Phys. Rev. Lett. 112, 218302 (2014).

[19] I. Williams, E. C. Oğuz, P. Bartlett, H. Löwen, and C. Patrick Royall, J. Chem. Phys. 142, 024505 (2015).

[20] K. Nygård, S. Sarman, K. Hyltegren, S. Chodankar, E. Perret, J. Buitenhuis, J. F. van der Veen, and R. Kjellander, Phys. Rev. X 6, 011014 (2016).

[21] B. Zhang and X. Cheng, Phys. Rev. Lett. 116, 098302 (2016).

[22] D. F. Kienle and T. L. Kuhl, Phys. Rev. Lett. 117, 036101 (2016).

[23] M. Lippmann, O. H. Seeck, A. Ehnes, K. Nygård, F. Bertram, and A. Ciobanu, J. Phys. Chem. Lett. 10, 1634 (2019).

[24] Y. Rosenfeld, M. Schmidt, H. Löwen, and P. Tarazona, Phys. Rev. E 55, 4245 (1997).

[25] C. Grodon, M. Dijkstra, R. Evans, and R. Roth, Mol. Phys. 103, 3009 (2005). 
[26] E. C. Oğuz, R. Messina, and H. Löwen, J. Phys.: Condens. Matter 21, 424110 (2009).

[27] T. Fehr and H. Löwen, Phys. Rev. E 52, 4016 (1995).

[28] G. Rodríguez and L. Vicente, Mol. Phys. 87, 213 (1996).

[29] D. Frydel and S. A. Rice, Phys. Rev. E 71, 041403 (2005).

[30] J. Mittal, J. R. Errington, and T. M. Truskett, J. Phys. Chem. B 111, 10054 (2007).

[31] G. Goel, W. P. Krekelberg, M. J. Pond, J. Mittal, V. K. Shen, J. R. Errington, and T. M. Truskett, J. Stat. Mech. (2009) P04006.

[32] S. H. Krishnan and K. G. Ayappa, Phys. Rev. E 86, 011504 (2012).

[33] T. S. Ingebrigtsen, J. R. Errington, T. M. Truskett, and J. C. Dyre, Phys. Rev. Lett. 111, 235901 (2013).

[34] S. Saw and C. Dasgupta, J. Chem. Phys. 145, 054707 (2016).

[35] P. Scheidler, W. Kob, and K. Binder, J. Phys. Chem. B 108, 6673 (2004).

[36] M. Spannuth and J. C. Conrad, Phys. Rev. Lett. 109, 028301 (2012).

[37] M. Fasolo and P. Sollich, Phys. Rev. Lett. 91, 068301 (2003).

[38] E. Zaccarelli, C. Valeriani, E. Sanz, W. C. K. Poon, M. E. Cates, and P. N. Pusey, Phys. Rev. Lett. 103, 135704 (2009).

[39] M. Campo and T. Speck, J. Stat. Mech. (2016) 084007

[40] P. K. Bommineni, N. R. Varela-Rosales, M. Klement, and M. Engel, Phys. Rev. Lett. 122, 128005 (2019).

[41] M. R. Hitchcock and C. K. Hall, J. Chem. Phys. 110, 11433 (1999).

[42] U. R. Pedersen, T. B. Schrøder, and J. C. Dyre, Phys. Rev. Lett. 120, 165501 (2018).

[43] K. Sandomirski, E. Allahyarov, H. Löwen, and S. U. Egelhaaf, Soft Matter 7, 8050 (2011).

[44] J. R. Espinosa, C. Vega, C. Valeriani, D. Frenkel, and E. Sanz, Soft Matter 15, 9625 (2019).

[45] B. J. Alder and T. E. Wainwright, J. Chem. Phys. 27, 1208 (1957).

[46] D. Rapaport, J. Comput. Phys. 34, 184 (1980).

[47] M. N. Bannerman, R. Sargant, and L. Lue, J. Comput. Chem. 32, 3329 (2011).

[48] D. Gazzillo and G. Pastore, Chem. Phys. Lett. 159, 388 (1989).
[49] A. Ninarello, L. Berthier, and D. Coslovich, Phys. Rev. X 7, 021039 (2017).

[50] L. Berthier, E. Flenner, C. J. Fullerton, C. Scalliet, and M. Singh, J. Stat. Mech. (2019) 064004.

[51] D. R. Nelson and B. I. Halperin, Phys. Rev. B 19, 2457 (1979).

[52] J. Chakrabarti and H. Löwen, Phys. Rev. E 58, 3400 (1998).

[53] P. N. Pusey, E. Zaccarelli, C. Valeriani, E. Sanz, W. C. K. Poon, and M. E. Cates, Philos. Trans. R. Soc., A 367, 4993 (2009).

[54] Y. Rosenfeld, Phys. Rev. Lett. 63, 980 (1989).

[55] R. Roth, J. Phys.: Condens. Matter 22, 063102 (2010).

[56] D. M. Heyes and A. Santos, J. Chem. Phys. 148, 214503 (2018).

[57] J. G. Kirkwood, J. Chem. Phys. 18, 380 (1950).

[58] J. A. Barker, Lattice Theories of the Liquid State (Pergamon, New York, 1963), Vol. 1.

[59] A. Bonissent, P. Pieranski, and P. Pieranski, Philosophical Magazine A 50, 57 (1984).

[60] J. J. Salacuse and G. Stell, J. Chem. Phys. 77, 3714 (1982).

[61] R. P. Sear, Europhys. Lett. 44, 531 (1998).

[62] P. Sollich, P. B. Warren, and M. E. Cates, in Advances in Chemical Physics, edited by I. Prigogine and S. A. Rice (Wiley, Hoboken, NJ, 2001), pp. 265-336.

[63] P. Pieranski, L. Strzelecki, and B. Pansu, Phys. Rev. Lett. 50, 900 (1983).

[64] D. H. Van Winkle and C. A. Murray, Phys. Rev. A 34, 562 (1986).

[65] R. Bubeck, C. Bechinger, S. Neser, and P. Leiderer, Phys. Rev. Lett. 82, 3364 (1999).

[66] R. Klaver and C. Schroën, Modeling Food Processing Operations, Woodhead Publishing Series in Food Science, Technology and Nutrition (Woodhead Publishing, Cambridge, UK, 2015), pp. 211-233.

[67] Y. L. Wu, D. Derks, A. van Blaaderen, and A. Imhof, Proc. Natl. Acad. Sci. USA 106, 10564 (2009).

[68] S. Iacopini, T. Palberg, and H. J. Schöpe, Phys. Rev. E 79, 010601(R) (2009).

[69] B. Cabane, J. Li, F. Artzner, R. Botet, C. Labbez, G. Bareigts, M. Sztucki, and L. Goehring, Phys. Rev. Lett. 116, 208001 (2016).

[70] P. C. Ohara, D. V. Leff, J. R. Heath, and W. M. Gelbart, Phys. Rev. Lett. 75, 3466 (1995). 